\section{Development and Validation of an Adaptive Method to Generate High-Resolution Quadrature Encoder Signals}

\author{
Erva Ulu \\ Graduate Research Assistant \\ Mem. ASME \\ Department of Mechanical Engineering, \\ Bilkent University, \\ Ankara 06800, Turkey \\ e-mail: erva@bilkent.edu.tr

\section{Nurcan Gecer Ulu} \\ Graduate Research Assistant \\ Mem. ASME \\ Department of Mechanical Engineering, \\ Bilkent University, \\ Ankara 06800, Turkey \\ e-mail: ulu@bilkent.edu.tr
}

\section{Melih Cakmakci}

Assistant Professor

Mem. ASME

Department of Mechanical Engineering,

Bilkent University,

Ankara 06800, Turkey

e-mail: melihc@bilkent.edu.tr

This paper presents a new method to increase the measurement resolution of quadrature encoders. The method contains an adaptive signal correction step and a signal interpolation step. Measured encoder signals contain imperfections including amplitude differences, mean offsets, and quadrature phase shift errors. With the proposed method, these errors are first corrected by using recursive least squares (RLS) estimation with exponential forgetting and resetting. Then, the corrected signals are interpolated to higher order sinusoids using a quick access look-up table generated offline. The position information can be obtained with the conversion of these high-order sinusoids to binary pulses and counting the zero crossings. Using the method presented here, a $10 \mathrm{~nm}$ measurement resolution is obtained using an encoder with $1 \mu m$ off-the-shelf resolution. Validation of the method and the practical limitations are also presented. Further increase in the resolution can be achieved by minimizing the effects of the electrical noise. [DOI: 10.1115/1.4026315]

\section{Introduction}

Applications of the precision motion control systems have been steadily increasing with the increasing demand for micro/nano technology related equipment [1-4]. Performance of a feedback based motion control system depends strongly on the precision and the resolution that can be obtained from its sensor since it provides the measured position information. Minimum achievable resolution of an optical encoder by decreasing the pitch of its scale

Contributed by the Dynamic Systems Division of ASME for publication in the Journal of Dynamic Systems, Measurement, and Control. Manuscript received September 27, 2012; final manuscript received December 14, 2013; published online February 19, 2014. Assoc. Editor: Srinivasa M. Salapaka. grating is limited by the available manufacturing technologies $[5,6]$. With the technologies of today, commercially available linear optical encoders may be produced with $0.512 \mu \mathrm{m}$ scale grating which corresponds to $0.128 \mu \mathrm{m}$ optical resolution.

Further improvement of the encoder resolution is possible by using signal processing (noise reduction and correction) and interpolation methods. Most interpolation methods assume ideal encoder signals with a quadrature phase difference between them. However, encoder signals usually contain some errors due to manufacturing tolerances of their scales, assembly problems, external environment conditions, and electrical grounding problems. Interpolation errors occur while extracting the position information from encoder signals distorted with such errors. Therefore, these errors have to be corrected before an interpolation method is applied.

Many different approaches have been developed to correct the distorted encoder signals containing amplitude errors, mean offsets, and quadrature phase shift errors. In Ref. [7], errors in encoder signal pairs are determined effectively using least squares minimization. Then, the signal is corrected based on the calculated error values. Since the correction parameters are calculated offline, this method does not offer effective compensation when the error varies throughout the motion as observed in Refs. [5] and [8]. In Ref. [9], a gradient search method is used to calculate the correction parameters online. The performance of this method at low frequencies and in the presence of signal noise may be poor [10]. In Ref. [6], the sinusoidal signals are obtained from a linear optical encoder by using an adaptive approach based on radial basis functions. Although high resolutions can be achieved with this method, encoder changes and changes in the environmental conditions require a new training period. In Ref. [5], high-order sinusoids from encoder signals are obtained and stored in a look-up table to achieve high resolution. In Ref. [11], a sine-cosine interpolation using logic gates and comparators is proposed. In Ref. [12], encoder signals are interpolated by using a digital signal processor and an analog-to-digital converter. This interpolation approach require additional hardware to obtain high resolution from the encoder and its applicability to typical servo controllers with a digital incremental encoder interface is limited [6].

In this paper, a new adaptive approach to obtain high resolution position information based on the measured encoder signals is presented. Our motivation is to develop a robust and an efficient real-time interpolation algorithm that can be used in high precision modular positioning systems [13]. Main contributions of the work presented here can be given as the adaptive characteristics of the encoder signal correction process and the simplicity of the interpolation step in terms of real-time computing performance. With the adaptive nature of the signal correction step, significant deviations in the encoder signals can be compensated and the method can be applied on different sinusoidal encoders without any parameter changes. These features also increase our method's applicability to multi-axis positioning systems requiring no changes in the algorithm [14].

The remainder of this paper is organized as follows: In Sec. 2, the developed method is discussed in detail. Section 3 presents the validation experiments with different resolutions including the best resolution we have obtained. Limitations on the proposed method due to some practical constraints are given in Sec. 4. Lastly, conclusions and future work are discussed in Sec. 5.

\section{Overview of the Proposed Approach}

The method proposed in this paper is composed of two main steps: (1) correction of signal errors and (2) interpolation of the corrected signals. These steps are labeled as Steps 1 and 2, respectively, in Fig. 1. In the correction step, corrected quadrature signals $u_{1}$ and $u_{2}$ are calculated from measured encoder signals $\hat{u}_{1}$ and $\hat{u}_{2}$. After the correction step is completed, an index number, $i$, calculated and corrected signals are mapped to high-order sinusoids, $u_{1 n}$ and $u_{2 n}$, from a look-up table during the interpolation 


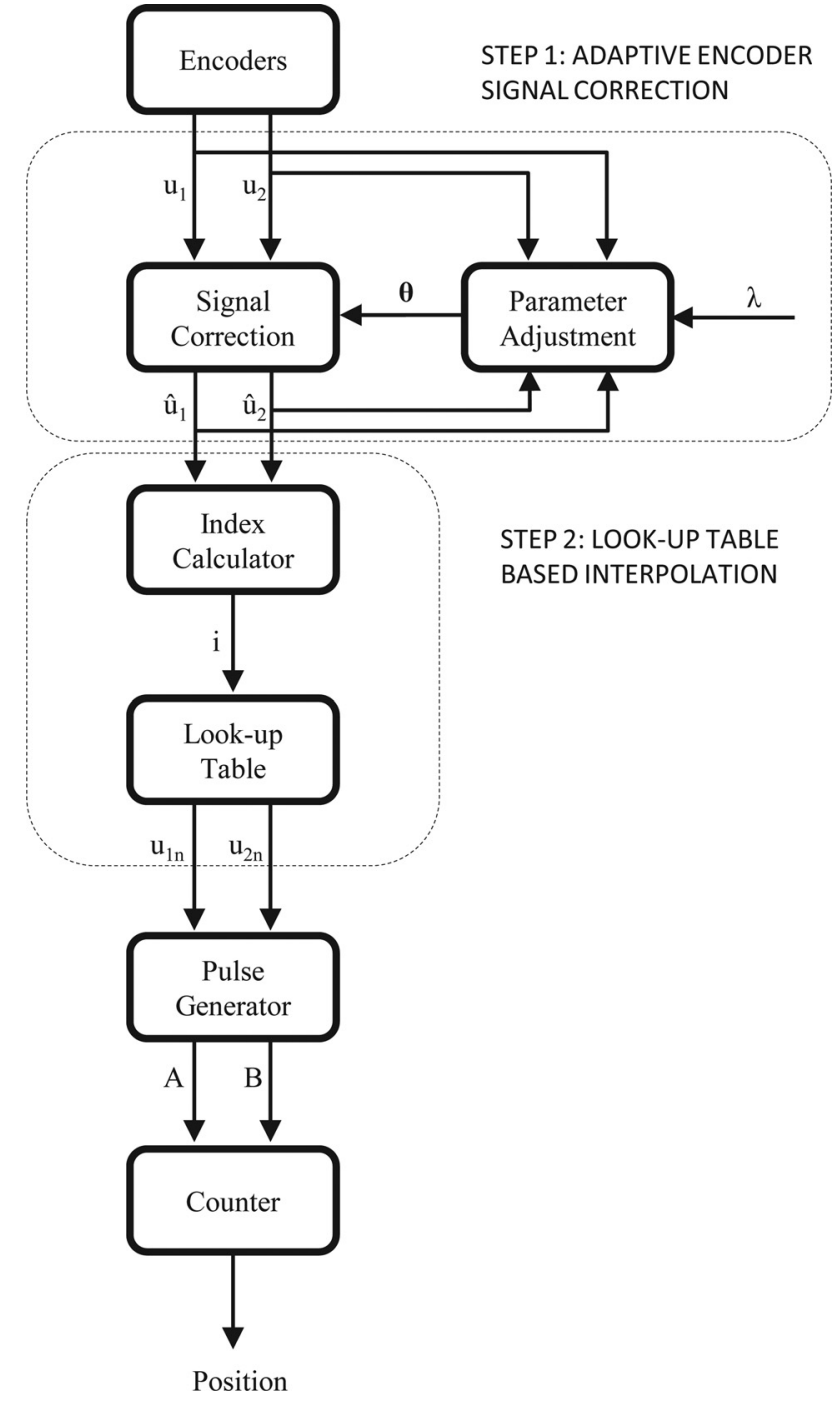

Fig. 1 General flow diagram of the proposed approach

step. A pulse generator is used to generate the quadrature binary pulses using these high-order sinusoids. Finally, the position information is calculated by detecting the zero crossings of these binary pulses.

In the next three subsections, Steps 1 and 2 and the pulse generation and counting parts of the algorithm shown in Fig. 1 are explained in detail.

2.1 Adaptive Encoder Signal Correction (Step 1). Measured signals from the encoder should be corrected before the interpolation step in order to avoid large errors. Common errors affecting the quadrature encoder signals are the amplitude $\left(A_{1}\right.$ and $\left.A_{2}\right)$ difference, the mean offsets $\left(m_{1}\right.$ and $\left.m_{2}\right)$ and the quadrature phase shift $(\phi)$ errors as shown in Fig. 2.

In some applications, it is possible to have a constant signal error all the way through the motion of the system. In such cases, the error can be compensated using offline correction methods as presented in Refs. [5] and [7]. When the encoder alignment is not performed effectively or the system has a long movement range, corresponding signal errors vary throughout the motion. Adaptive correction approaches may be used to compensate these varying errors to obtain higher encoder resolutions.

In this study, a recursive least squares estimation with exponential forgetting and resetting is used to adjust correction parameters

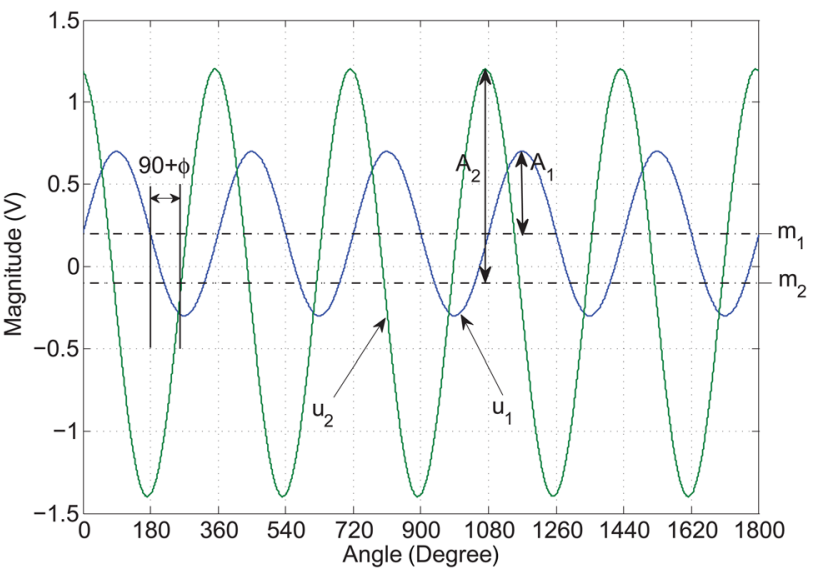

Fig. 2 Measured encoder signals with imperfections

online. An ideal set of quadrature encoder signals, $u_{1 i}$ and $u_{2 i}$, can be expressed as

$$
\begin{aligned}
& u_{1 i}=A \cos \alpha \\
& u_{2 i}=A \sin \alpha
\end{aligned}
$$

where $A$ is the amplitude and $\alpha$ is the instantaneous angle of the signals with a phase difference of $\pi / 2$ radians.

Choosing an ideal set of quadrature encoder signals with amplitude $A_{1}$, the relationship between the measured $\left(u_{1}\right.$ and $\left.u_{2}\right)$ and the ideal $\left(u_{1 i}\right.$ and $\left.u_{2 i}\right)$ encoder signals can be written as

$$
\begin{aligned}
& u_{1}=u_{1 i}+m_{1} \\
& u_{2}=\frac{1}{R}\left(A_{1} \cos (\alpha-\phi)\right)+m_{2}
\end{aligned}
$$

where $m_{1}$ and $m_{2}$ are the mean offset values, $\phi$ is the quadrature phase shift error, $A_{1}$ and $A_{2}$ are the amplitudes of measured encoder signals and $R$ is the gain ratio $\left(A_{1} / A_{2}\right)$ of the amplitudes. Using Eqs. (1) and (2), a least squares estimation formulation can be constructed as shown in Eqs. (3) and (4).

$$
\theta_{1} u_{1}^{2}+\theta_{2} u_{2}^{2}+\theta_{3} u_{1} u_{2}+\theta_{4} u_{1}+\theta_{5} u_{2}=1
$$

where

$$
\begin{aligned}
& \theta_{1}=\left(A_{1}^{2} \cos ^{2} \phi-m_{1}^{2}-R^{2} m_{2}^{2}-2 R m_{1} m_{2} \sin \phi\right)^{-1} \\
& \theta_{2}=\theta_{1} R^{2} \\
& \theta_{3}=2 \theta_{1} R \sin \phi \\
& \theta_{4}=-2 \theta_{1}\left(m_{1}+R m_{2} \sin \phi\right) \\
& \theta_{5}=-2 \theta_{1} R\left(R m_{2}+m_{1} \sin \phi\right)
\end{aligned}
$$

It is possible to calculate $\theta_{i}(i=1,2, \ldots, 5)$ values offline. Since we assume that the error changes dynamically, all parameters used in Eq. (3) are functions of time as shown below

$$
\begin{aligned}
& \varphi_{1}(t) \theta_{1}(t)+\varphi_{2}(t) \theta_{2}(t)+\varphi_{3}(t) \theta_{3}(t)+\varphi_{4}(t) \theta_{4}(t) \\
& +\varphi_{5}(t) \theta_{5}(t)=\boldsymbol{\varphi}^{T}(t) \boldsymbol{\theta}(t)=1
\end{aligned}
$$

where superscript $T$ denotes the transpose of a matrix, $t$ is the time index, $\theta_{i}^{\prime} s$ are parameters to be determined, and $\varphi_{i}^{\prime} s$ are calculated functions from the measured encoder signal values (i.e., $u_{i} \mathrm{~s}$ ).

The objective of an RLS estimation algorithm is to determine the parameters so that Eq. (5) is satisfied with minimum error. For this purpose, a standard error-based cost function given in Eq. (6) can be used. 


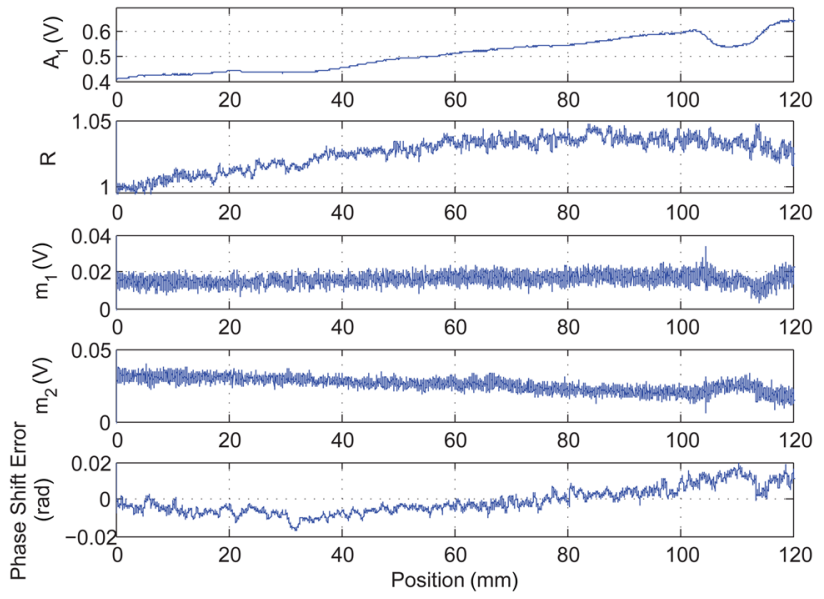

Fig. 3 Encoder signal parameters recorded through $120 \mathrm{~mm}$ motion of the single axis slider

$$
V(\boldsymbol{\theta}, t)=\frac{1}{2} \sum_{k=1}^{t} \lambda^{t-k}\left(1-\boldsymbol{\varphi}^{T}(k) \boldsymbol{\theta}\right)^{2}
$$

In Eq. (6), $k$ is the index and $\lambda$ is a forgetting factor $(0<\lambda \leq 1)$. By selecting the value of $\lambda$, the contribution of the previous data points to the current value of the cost function can be adjusted. Then, a recursive parameter adjustment law which minimizes the cost function shown in Eq. (6) can be given as shown below:

$$
\begin{aligned}
& \boldsymbol{\theta}(t)=\boldsymbol{\theta}(t-1)+\boldsymbol{K}(t)\left(1-\boldsymbol{\varphi}^{T}(t) \boldsymbol{\theta}(t-1)\right) \\
& \boldsymbol{K}(t)=\boldsymbol{P}(t-1) \boldsymbol{\varphi}(t)\left(\lambda+\boldsymbol{\varphi}^{T}(t) \boldsymbol{P}(t-1) \boldsymbol{\varphi}(t)\right)^{-1} \\
& \boldsymbol{P}(t)=\left(\boldsymbol{I}-\boldsymbol{K}(t) \boldsymbol{\varphi}^{T}(t)\right) \boldsymbol{P}(t-1) / \lambda
\end{aligned}
$$

where $\boldsymbol{I}$ is identity matrix and $\boldsymbol{P}$ is a nonsingular matrix. $\boldsymbol{P}$ can be chosen as $\boldsymbol{P}=\kappa \boldsymbol{I}$, where $\kappa$ is a large number. The parameter adjustment mechanism described in Eq. (7) is known as the recursive least squares estimation with exponential forgetting [15]. It should be noted that the overall performance of the correction algorithm changes with the selection of the forgetting factor, $\lambda$, the initial $P$ matrix and the resetting period under different noise and disturbance effects.

From this point forward time index $t$ will be dropped from the formulations for simplicity. Given $\boldsymbol{\theta}$, the encoder signal correction parameters (i.e., $A_{1}, R, m_{1}, m_{2}$, and $\phi$ ) can be calculated as shown in Eq. (8) using the formulation in Eq. (4)

$$
\begin{aligned}
\phi & =\arcsin \left(\theta_{3} / \sqrt{4 \theta_{1} \theta_{2}}\right) \\
R & =\sqrt{\theta_{2} / \theta_{1}} \\
m_{1} & =\left(2 \theta_{2} \theta_{4}-\theta_{5} \theta_{3}\right) /\left(\theta_{3}^{2}-4 \theta_{1} \theta_{2}\right) \\
m_{2} & =\left(2 \theta_{1} \theta_{5}-\theta_{4} \theta_{3}\right) /\left(\theta_{3}^{2}-4 \theta_{1} \theta_{2}\right) \\
A_{1} & =\sqrt{\frac{4 \theta_{2}\left(1+\theta_{1} m_{1}^{2}+\theta_{2} m_{2}^{2}+\theta_{3} m_{1} m_{2}\right)}{4 \theta_{1} \theta_{2}-\theta_{3}^{2}}}
\end{aligned}
$$

Then the corrected quadrature signals, $\hat{u}_{1}$ and $\hat{u}_{2}$ can be calculated as shown in below equation:

$$
\begin{aligned}
& \hat{u}_{1}=\frac{1}{A_{1}}\left(u_{1}-m_{1}\right) \\
& \hat{u}_{2}=\frac{1}{A_{1} \cos \phi}\left(\left(u_{1}-m_{1}\right) \sin \phi+R\left(u_{2}-m_{2}\right)\right)
\end{aligned}
$$

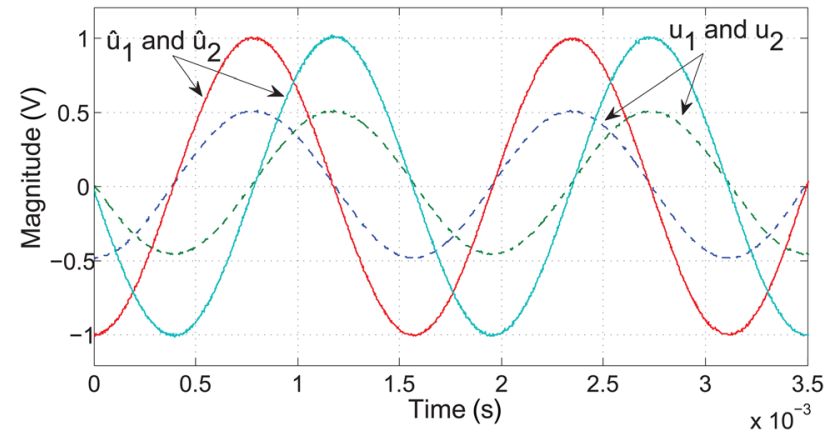

Fig. 4 Corrected and measured (actual) encoder signals

By applying the adjustment law presented in Eq. (7), correction parameters are updated at each iteration step recursively considering the effects of parameter values from the previous iterations. Hence, slow changes in the parameters can be covered efficiently. In Fig. 3, changes in the signal amplitude, gain ratio, mean offsets, and phase shift errors recorded during the $120 \mathrm{~mm}$ motion of our single axis slider are shown. These traces show both slowly and suddenly varying parameter value regions. To compensate for the cases where sudden parameter value changes occur, the $\boldsymbol{P}$ matrix given in Eq. (7) is reset periodically to its initial value (i.e., to $\kappa \boldsymbol{I}$ ). As a result of resetting, the parameter estimate is updated with a larger step size so that significant changes in the parameters can be estimated properly as also explained in Ref. [15]. Resulting convergence properties of parameters $m_{1}, m_{2}, A_{1}, R$, and $\theta$ are also important for the performance of the interpolation algorithm. Based on our studies for representative cases, our system's convergence rate is about 90 samples at the worst case while remaining within the range of variations observed in Fig. 3 for each parameter. Illustration of the encoder signals obtained before and after the correction step using RLS estimation with exponential forgetting and resetting is given in Fig. 4.

2.2 Signal Interpolation (Step 2). As the second step of the method presented in this paper, a look-up table based interpolation procedure is developed. Calculation of the higher order sinusoids can be a tedious operation and may reduce the accuracy and the real time performance of the interpolation process [5]. When the signals obtained after the adaptive signal correction step are sufficiently close to the ideal sinusoidal signal values, a quick access look-up table using the actual numerical values of high-order sinusoids can be generated offline and used. This approach is also easy to implement in practice and applicable to different encoders and/or operating conditions.

The index calculator shown in Fig. 1, uses the values of the corrected signals, $\hat{u}_{1}$ and $\hat{u}_{2}$, as inputs and generates an index number, $i$. Using this number as the address (i.e., row number) on the table, values of $n$th order sinusoids, $u_{1 n}$ and $u_{2 n}$, corresponding to $\hat{u}_{1}$ and $\hat{u}_{2}$ are calculated from the look-up table. The look-up table is constructed by dividing each octant (i.e., an interval of $\pi / 4 \mathrm{rad}$ ) to a set of $n$th order sinusoidal signals, $\cos (n \alpha)$ and $\sin (n \alpha)$, into $N$ samples leading $8 \mathrm{~N}$ samples over one period of a high-order sinusoid. Then, values of these samples are stored in the look-up table prior to the operation. An index based look-up table for an $n$th order interpolation is given in Table 1 .

In order to map the corrected signals to the $n$th order sinusoids using the look-up table, the corresponding index number should be calculated. As also observed by researchers in Ref. [5], when the index calculation is based on just one signal $\left(\hat{u}_{1}\right.$ or $\left.\hat{u}_{2}\right)$ poor resolution will be obtained around $\hat{u}_{1} \approx 1$ or $\hat{u}_{2} \approx 1$ due to the highly nonlinear relationship between the magnitude and the angle of the signals in these regions. Using large $N$ values may solve this problem. However large $\mathrm{N}$ will increase the size of the 
Table 1 A generic look-up table

\begin{tabular}{lcc}
\hline \hline Index & $\cos (n \alpha)$ & $\sin (n \alpha)$ \\
\hline 0 & 1 & 0 \\
1 & $\cos \left(\frac{n \pi}{8 N}\right)$ & $\sin \left(\frac{n \pi}{8 N}\right)$ \\
2 & $\cos \left(\frac{n \pi}{4 N}\right)$ & $\sin \left(\frac{n \pi}{4 N}\right)$ \\
$i$ & $(\vdots n \pi)$ & $:$ \\
$8 N$ & $\cos \left(\frac{n i \pi}{8 N}\right)$ & $\sin \left(\frac{n i \pi}{8 N}\right)$ \\
$:$ & $:$ & $:$ \\
\hline \hline
\end{tabular}

Table 2 Index calculation table

\begin{tabular}{|c|c|c|c|}
\hline Sign & Magnitude & $i$ & $\alpha$ \\
\hline $\operatorname{sign}\left(\hat{u}_{1}\right)>0$ & $\hat{u}_{2} \geq \sin \left(\frac{\pi}{4}\right)$ & $\frac{\hat{u}_{1} N}{\sin (\pi / 4)}$ & $\left(0, \frac{\pi}{4}\right]$ \\
\hline $\operatorname{sign}\left(\hat{u}_{2}\right) \geq 0$ & $\hat{u}_{1}>\sin \left(\frac{\pi}{4}\right)$ & $2 N-\frac{\hat{u}_{2} N}{\sin (\pi / 4)}$ & $\left(\frac{\pi}{4}, \frac{\pi}{2}\right]$ \\
\hline $\operatorname{sign}\left(\hat{u}_{2}\right)<0$ & $\hat{u}_{1} \geq \sin \left(\frac{\pi}{4}\right)$ & $2 N-\frac{\hat{u}_{2} N}{\sin (\pi / 4)}$ & $\left(\frac{\pi}{2}, \frac{3 \pi}{4}\right]$ \\
\hline $\operatorname{sign}\left(\hat{u}_{1}\right) \geq 0$ & $\hat{u}_{2}<-\sin \left(\frac{\pi}{4}\right)$ & $4 N-\frac{\hat{u}_{1} N}{\sin (\pi / 4)}$ & $\left(\frac{3 \pi}{4}, \pi\right]$ \\
\hline $\operatorname{sign}\left(\hat{u}_{1}\right)<0$ & $\hat{u}_{2} \leq-\sin \left(\frac{\pi}{4}\right)$ & $4 N-\frac{\hat{u}_{1} N}{\sin (\pi / 4)}$ & $\left(\pi, \frac{5 \pi}{4}\right]$ \\
\hline $\operatorname{sign}\left(\hat{u}_{2}\right) \leq 0$ & $\hat{u}_{1}<-\sin \left(\frac{\pi}{4}\right)$ & $6 N+\frac{\hat{u}_{2} N}{\sin (\pi / 4)}$ & $\left(\frac{5 \pi}{4}, \frac{3 \pi}{2}\right]$ \\
\hline $\operatorname{sign}\left(\hat{u}_{2}\right)>0$ & $\hat{u}_{1} \leq-\sin \left(\frac{\pi}{4}\right)$ & $6 N+\frac{\hat{u}_{2} N}{\sin (\pi / 4)}$ & $\left(\frac{3 \pi}{2}, \frac{7 \pi}{4}\right]$ \\
\hline $\operatorname{sign}\left(\hat{u}_{1}\right) \leq 0$ & $\hat{u}_{2}>\sin \left(\frac{\pi}{4}\right)$ & $8 N+\frac{\hat{u}_{1} N}{\sin (\pi / 4)}$ & $\left(\frac{7 \pi}{4}, 2 \pi\right]$ \\
\hline
\end{tabular}

look-up table increasing the computational burden. As a more computationally efficient alternative, the index number may be calculated using one of the signals, $\hat{u}_{1}$ or $\hat{u}_{2}$, depending on which signal is outside of the poor resolution region. The poor resolution region to avoid for each signal is defined in below equation:

$$
\begin{aligned}
& \left|\hat{u}_{1}\right|>\sin \left(\frac{\pi}{4}\right) \\
& \left|\hat{u}_{2}\right|>\sin \left(\frac{\pi}{4}\right)
\end{aligned}
$$

The index number, $i$, can be calculated by using the nearly linear relationship between the magnitude and the angle $\alpha$ outside the poor regions. The signs and magnitudes of $\hat{u}_{1}$ and $\hat{u}_{2}$ signals can be used to find the correct octant of the current angle. For example, for $0<\alpha \leq \pi / 4$, the conditions $\operatorname{sign}\left(\hat{u}_{1}\right)>0$ and $\hat{u}_{2} \geq \sin (\pi / 4)$ are valid. Since $\hat{u}_{2}$ is in the poor resolution region mentioned above, it is suitable to use the value of $\hat{u}_{1}$ to calculate the index value. Using the linear relationship between the magnitude of $\hat{u}_{1}$ and the angle, $\alpha$, index number, $i$, can be calculated as shown in below equation:

$$
i=\frac{\hat{u}_{1} N}{\sin (\pi / 4)}
$$

In Table 2, two conditions are defined to calculate the index number given signals $\hat{u}_{1}$ and $\hat{u}_{2}$. These are labeled as the sign and the

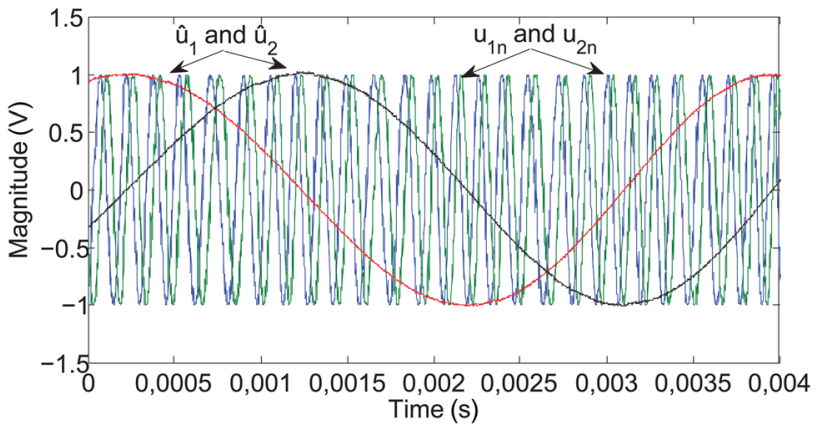

Fig. 5 Interpolation results for $n=25$

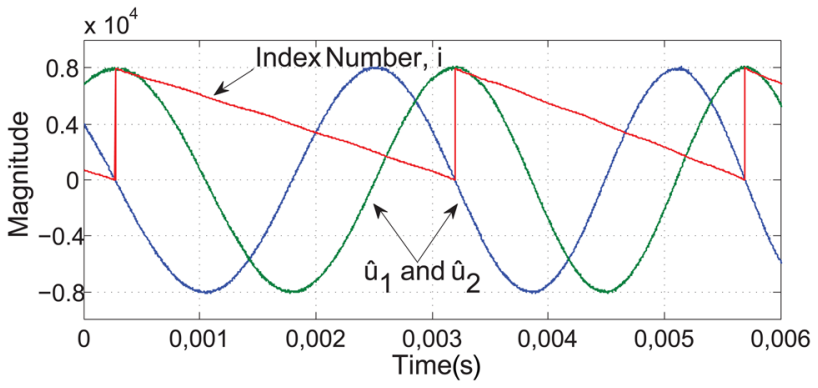

Fig. 6 Variation of index number for $n=25$

magnitude conditions. Once a row which satisfies both the sign and the magnitude conditions is identified, the corresponding formula is used to calculate the index value as described in the previous paragraph. Since the table is constructed by considering only the signs and magnitudes of the available encoder signals, it is easier to understand for analysis and implement in real-time compared to the alternatives reported in the literature. While using Eq. (11) or similar ones given in Table 2 for the other octants of sinusoids, index numbers obtained may not always be integers. For such cases the index value should be rounded to the closest integer value in order to read the correct value in the look-up table.

After using Table 2 for calculation of the index number and obtaining the corresponding values from the look-up table (Table 1), $n$th order sinusoidal signals can be obtained. Interpolation results for $n=25$ is given in Fig. 5 where $N$ is chosen as 1000 . For the same example, the calculated index number, $i$, and the corresponding corrected signals, $\hat{u}_{1}$ and $\hat{u}_{2}$, are shown together in Fig. 6. Amplitudes of both sinusoidal signals are set to 8000 intentionally to show the relationship between signal and the index number clearly for this figure. As expected, the index number varies from 0 to $8000(8 N)$ linearly in one period of signals.

2.3 Binary Pulse Generation. The interpolated encoder signals, $u_{1 n}$ and $u_{2 n}$, should be converted to binary pulses for calculating the position information. The position information can be obtained by counting the zero-crossings of these binary pulses. Although additional hardware can be used, this can be done as part of the algorithm by generating the $A$ and the $B$ binary pulses given in below equation:

$$
\begin{aligned}
& A=\left\{\begin{array}{ccc}
1 & \text { if } \quad & u_{1 n} \geq \varepsilon \\
-1 & \text { if } & u_{1 n} \leq-\varepsilon
\end{array}\right. \\
& B=\left\{\begin{array}{ccc}
1 & \text { if } \quad & u_{2 n} \geq \varepsilon \\
-1 & \text { if } & u_{2 n} \leq-\varepsilon
\end{array}\right.
\end{aligned}
$$

where $\varepsilon$ is a threshold value. With the proper selection of this threshold, undesired zero crossings can be eliminated. 


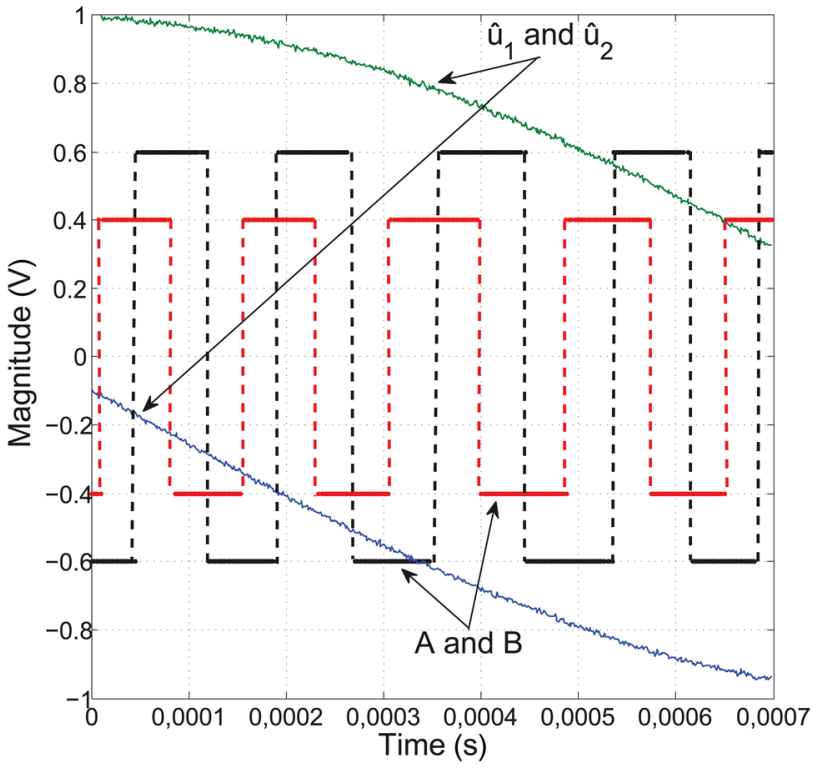

Fig. 7 Binary pulses obtained for $\mathrm{n}=\mathbf{2 5}$

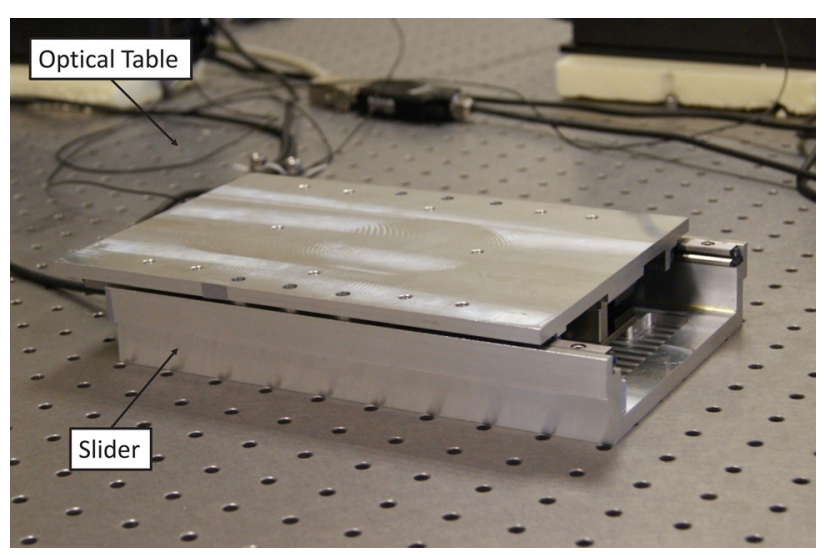

Fig. 8 Single axis slider

In Fig. 7, binary pulses generated using Eq. (12) are shown with the corrected analog signals $\left(\hat{u}_{1}\right.$ and $\left.\hat{u}_{2}\right)$ when the threshold, $\varepsilon$, is set to $0.05 \mathrm{~V}$. Amplitudes of binary pulses are set to 0.4 and 0.6 for $A$ and $B$, respectively, for presentation purposes.

\section{Validation and Closed-Loop Experiment Results}

In this section, the performance of the proposed encoder signal interpolation method using different resolutions is presented. The experiments are conducted on a single axis slider positioning system shown in Fig. 8. The slider is driven by a permanent magnet linear motor. A linear optical encoder with the original measurement resolution of $1 \mu \mathrm{m}$ is used as the position sensor. Travel range of the slider is $120 \mathrm{~mm}$. The sensor signals are received from the linear encoder attached to the slider. Then, the proposed method is applied in real-time to obtain high resolution position measurements for different cases.

In Figs. 9 and 10 the interpolation results with $n=50$ and $n=100$ are shown, respectively. In Fig. 5, interpolation with $n=25$ is also given. In these figures, interpolated signals and corrected signals are shown together to illustrate the performance of the presented method. All experiments are performed using $N=1000$ samples per octant.

In order to validate our interpolation method externally a test setup is prepared using a two-arm differential laser vibrometer with $3 \mathrm{~nm}$ measurement resolution. One of the laser arms is

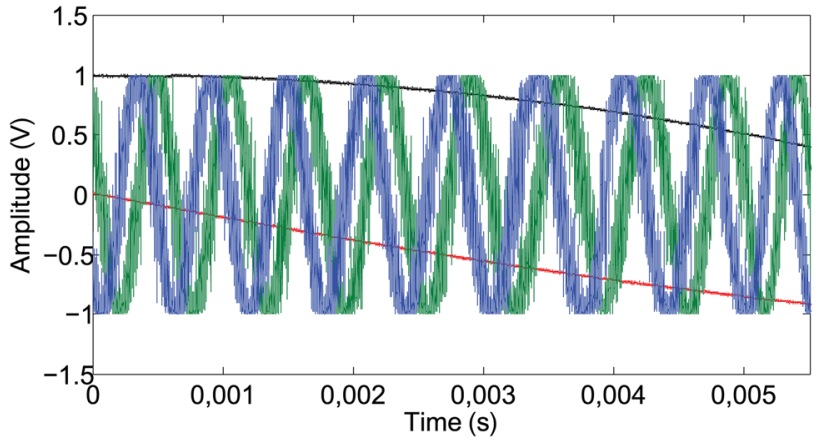

Fig. 9 Interpolation results for $\boldsymbol{n}=\mathbf{5 0}$

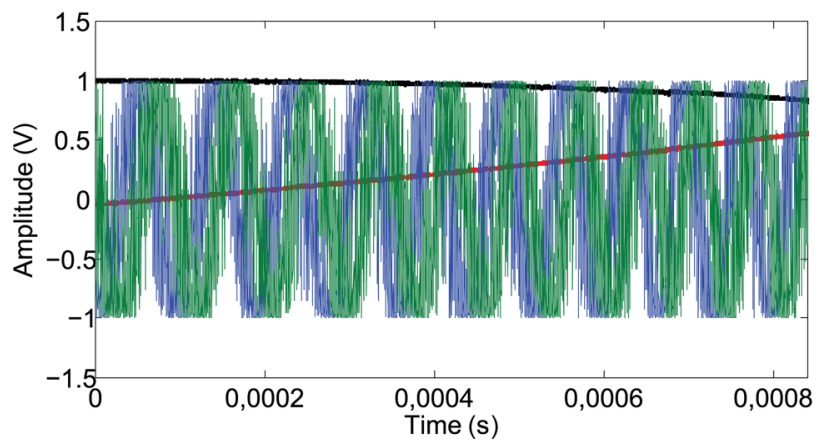

Fig. 10 Interpolation results for $n=100$

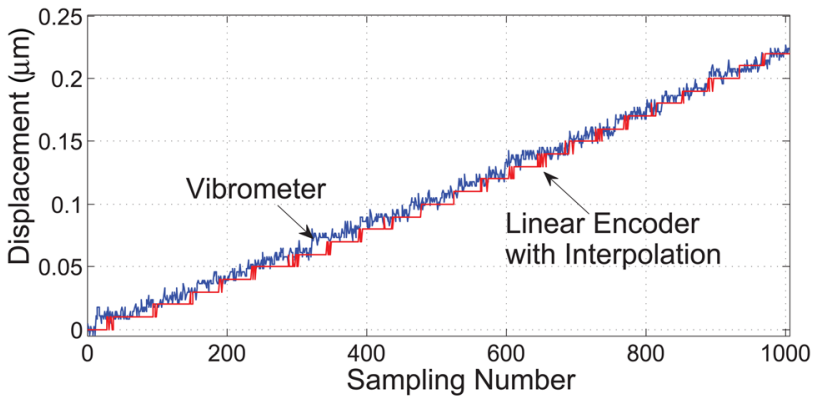

(a)

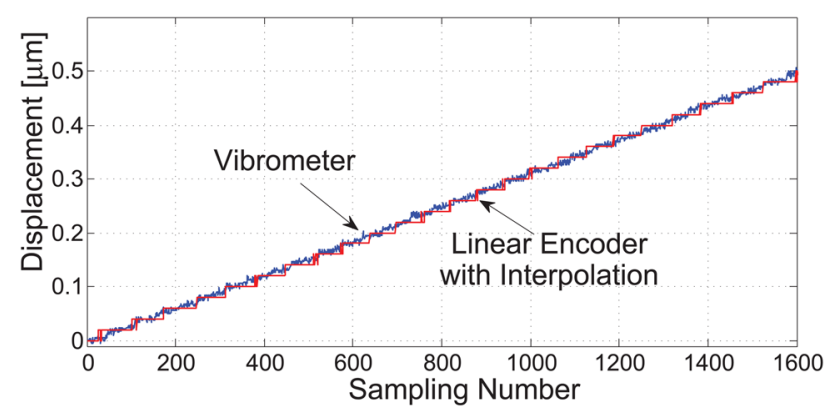

(a)

Fig. 11 Validation of the proposed method for (a) $n=100$ and (b) $n=50$

directed to the stationary part of the slider as the reference and the other arm is positioned to point at the moving part of the slider system. In Figs. 11(a) and 11(b), the displacement measurements obtained with the vibrometer and the linear encoder with interpolation are given together for $n=100, n=50$. These values correspond to measurement resolutions of $10 \mathrm{~nm}, 20 \mathrm{~nm}$, respectively. 


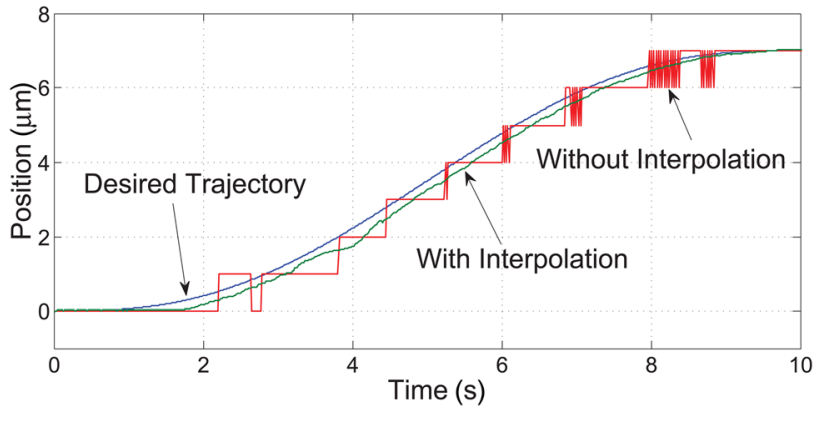

Fig. 12 Tracking performance of the single axis slider

For both cases, interpolation results match with the displacements measured with laser vibrometer. Root mean square (RMS) values for the measurement difference between the vibrometer and the linear encoder with interpolation are calculated as $7.2 \mathrm{~nm}$ and $8.3 \mathrm{~nm}$ for $n=100$ and $n=50$, respectively. Since these values are below the intended measurement resolutions, it can be concluded that the proposed method is validated successfully.

The positioning performance of the single axis slider system is evaluated to compare the cases with and without interpolation of the encoder signals. A conventional proportional-integralderivative (PID) controller is used as the feedback controller. For the test "with interpolation," $n=100$ is used so that the resolution of the measured position is $10 \mathrm{~nm}$. In Fig. 12, the tracking performance of the system for a reference input of $7 \mu \mathrm{m}$ is given. In order to compare the tracking errors better, the reference input is given as an S-curve. In this figure, the significantly improved tracking performance of the system at sub-micrometer level is observed clearly. For the test "without interpolation," RMS of the tracking error is calculated as $312.14 \mathrm{~nm}$. When the encoder resolution is increased to $10 \mathrm{~nm}$ using the interpolation method, the same error is reduced to $121.53 \mathrm{~nm}$.

The method presented here is also successfully implemented on a two axis positioning system achieving high-tracking and contouring accuracy. Details of this study can be found in Ref. [14].

\section{Limitations}

Application of the proposed method to generate high-resolution quadrature encoder signals may be limited in some cases due to practical constraints such as the sampling capabilities of the system, the velocity of slider and the signal noise.

Linear optical encoders generate sinusoidal signals with a period equal to the grating pitch of the encoder scale, $l_{p}$. With the implementation of the proposed method, $n$th order sinusoids with a period equal to $l_{p} / n$ are generated. Hence, when $N_{1}$ samples are recorded in one period of original first order sinusoidal encoder signal, interpolation number can be expressed as $n=N_{1} / N_{n}$, where $N_{n}$ is the number of samples in one period of $n$th order sinusoid. Similarly, the maximum interpolation number, $n_{\max }$, can then be calculated as $n_{\max }=N_{1}^{\max } / N_{n}^{\min }$, where $N_{1}^{\max }$ is the maximum number of samples that can be recorded in one period of the original encoder signals and $N_{n}^{\min }$ is the minimum number of samples to represent an $n$th order sinusoid. This calculation shows the minimum encoder resolution is limited by the sampling performance for the system.

Another constraint for the interpolation number is the velocity of the slider motion. Defining the loop time, $t_{l p}$, as the time required to process one sample of the encoder signal, the maximum interpolation number and maximum velocity of the motion, $v_{\max }$, are related as shown in below equation:

$$
n_{\max }=\frac{l_{p}}{v_{\max } N_{n} t_{l p}}
$$

The electrical noise affecting the encoder signals is also an important factor limiting the interpolation number. Sensitivity to noise increases with increasing $n$ as observed in Figs. 5, 9, and 10. In order to reach high encoder resolution using the proposed interpolation method, the noise in the encoder signals should be minimized by shielding, grounding and filtering. The effect of noise can also be eliminated with the proper selection of the threshold value, $\epsilon$, during the pulse generation task as previously mentioned.

\section{Conclusions}

In this paper, a new method to calculate high resolution position information from measured quadrature encoder signals is presented. The signal errors are corrected first by using a recursive least squares estimation with exponential forgetting and resetting. Due to the adaptive characteristics of the correction algorithm, dynamically changing errors can be compensated effectively. Then, these corrected signals are mapped to high-order sinusoids by using a computationally effective look up table based interpolation calculation. The external validation of the method using a differential laser vibrometer is presented. The effectiveness of the proposed method in real-time closed loop systems is illustrated with the experimental results. Limitations on the proposed method due to the practical constraints are also discussed.

Using the proposed method, $10 \mathrm{~nm}$ measurement resolution is obtained with an optical encoder having $1 \mu \mathrm{m}$ original (off-the-shelf) resolution. Applicability of the proposed method is proven with the implementation on single and two-axis positioning devices without requiring any additional hardware. Combining our interpolation method with a suitable controller, tracking performance of both single and multiple axis systems are increased significantly.

Although nanometer level resolutions are obtained with the presented method, its sensitivity to noise cannot be ignored for large interpolation numbers $(n>100)$. Our future work focuses on the reduction of sensitivity to noise by hardware and the improvements to our interpolation method with the inclusion of various stochastic signal models.

\section{Acknowledgment}

This research is sponsored by Scientific and Technical Research Council of Turkey (TUBITAK) through Project No: 110M251. Authors also would like to thank Dr. Sinan Filiz and undergraduate students Ersun Sozen and Oytun Ugurel for their support during the mechanical design of the slider system.

\section{References}

[1] Devasia, S., Eleftheriou, E. E., and Moheimani, R., 2007, “A Survey of Control Issues in Nanopositioning," IEEE Trans. Control Syst. Technol., 15(15), pp. $802-823$.

[2] Manske, E., Hausotte, T., Mastylo, R., Machleidt, T., Franke, K., and Jager, G., 2007, "New Applications of the Nanopositioning and Nanomeasuring Machine by Using Advanced Tactile and Non-Tactile Probes," Meas. Sci. Technol., 18(2), pp. 520-527.

[3] Lihua, L., Yingchun, L., Yongfeng, G., and Akira, S., 2010, "Design and Testing of a Nanometer Positioning System," ASME J. Dyn. Syst., Meas., Control, 132(2), p. 021011.

[4] Pang, C. K., Guo, G., Chen, B. M., and Lee, T. H., 2006, "Self-Sensing Actuation for Nanopositioning and Active-Mode Damping in Dual Stage HDDs," IEEE/ASME Trans. Mechatronics, 11(3), pp. 328-338.

[5] Tan, K. K., Zhou, H. X., and Lee, T. H., 2002, "New Interpolation Method for Quadrature Encoder Signals," IEEE Trans. Instrum. Meas., 51(5), pp. 1073-1079.

[6] Tan, K. K., and Tang, K. Z., 2005, "Adaptive Online Correction and Interpolation of Quadrature Encoder Signals Using Radial Basis Functions," IEEE Trans. Control Syst. Technol., 13(3), pp. 370-377.

[7] Heydemann, P. L. M., 1981, "Determination and Correction of Quadrature Fringe Measurement Errors in Interferometers," Appl. Opt., 20(19), pp. 3382-3384.

[8] Birch, K. P., 1990, "Optical Fringe Subdivision With Nanometric Accuracy," Precis. Eng., 12(4), pp. 195-198.

[9] Balemi, S., 2005, "Automatic Calibration of Sinusoidal Encoder Signals," 16th IFAC World Congress, Prague, Czech Republic, July 4-8. 
[10] Hoang, H. V., and Jeon, J. W., 2011, "An Efficient Approach to Correct the Signals and Generate High-Resolution Quadrature Pulses for Magnetic Encoders," IEEE Trans. Ind. Electron., 58(8), pp. 3634-3646.

[11] Cheung, N. C., 1999, "An Innovative Method to Increase the Resolution of Optical Encoders in Motion Servo Systems," IEEE International Conference on Power Electronics and Drive Systems (PEDS '99), Hong Kong, July 27-29, pp. 797-802.

[12] Madni, A. M., Jumper, M., and Malcolm, T., 2001, "An Absolute HighPerformance, Self-Calibrating Optical Rotary Positioning System," Proceedings of IEEE Aerospace Conference, Big Sky, MT, March 10-17, Vol. 5, pp. $2363-2373$
[13] Ulu, E., Gecer-Ulu, N., and Cakmakci, M., 2012, "Adaptive Correction and Look-Up Table Based Interpolation of Quadrature Encoder Signals," ASME Dynamic Systems and Control Conference, Fort Lauderdale, FL, October 17-19, ASME Paper No. DSCC2012MOVIC2012-8661.

[14] Gecer-Ulu, N., Ulu, E., and Cakmakci, M., 2012, "Learning Based CrossCoupled Control for Multi-Axis High Precision Positioning Systems," ASME Dynamic Systems and Control Conference, Fort Lauderdale, FL, October 17-19, ASME Paper No. DSCC2012-MOVIC2012-8660.

[15] Astrom, K. J., and Wittenmark, B., 1995, Adaptive Control, 2nd ed., Dover Publications, Mineola, NY, pp. 42-56. 